\title{
Caracterización del docente en la educación virtual: considera- ciones para la Bibliotecología
}

\author{
Roberto Garduño Vera *
}

Artículo recibido:

30 de agosto de 2006.

Artículo aceptado:

1 de mayo de 2007.

\section{RESUMEN}

Los primeros años del siglo XXI evidencian que la enseñanza de las disciplinas en sus distintos niveles y modalidades ha sido afectada por los progresos derivados de la investigación científica y tecnológica. La innovación educativa, la privatización y comercialización del conocimiento y la demanda social creciente de propuestas educativas, han ocasionado que las instituciones dedicadas a la educación incidan en programas que faciliten el desarrollo sustentable relacionado con la prestación de servicios educativos. Así, la educación virtual como una alternativa que facilite tal propósito, hace uso de la información, de los contenidos (objetos de aprendizaje) y de los medios de comunicación

* Centro Universitario de Investigaciones Bibliotecológicas de la UNAM, México. garduno@servidor.unam.mx

INVESTIGACIÓN BIBLIOTECOLÓGICA, Vol. 21, Núm. 43, julio/diciembre, 2007, México, ISSN: 0187-358X. pp. 157-183 
que propician el diálogo académico con la intención de apoyar el proceso de comunicación entre los sujetos del acto educativo a distancia. Esto ha acelerado la urgencia de discutir y definir con mayor precisión cuáles son las características requeridas del docente que se dedicará a la educación virtual, por lo tanto, que en la actualidad se ha convertido en un asunto discutido por los más diversos grupos interdisciplinarios. Este documento reflexiona principalmente en torno a los siguientes asuntos: caracterización y orientación definitoria del docente que se involucrará con programas de educación virtual; las tutorías para la educación virtual en Bibliotecología, el autor-tutor de objetos de aprendizaje, y la función de los estándares educativos en el desarrollo de objetos de aprendizaje.

Palabras clave: Tutoría y educación virtual; Desarrollo de objetos de aprendizaje; Estándares educativos; Educación virtual en bibliotecología.

\section{ABSTRACT \\ Teacher Characterization in virtual education: Delibe- rations for Library Science \\ Roberto Garduño Vera}

Evidently, teaching in this century has been affected by the progress achieved in scientific and technological research. Educational innovation, privatization and commercialization of knowledge and a growing social demand for educational proposals have meant that institutions dedicated to education, have promoted programs which facilitate the sustainable development of educational services. Virtual education, as an alternative for this proposal, propitiates academic dialog among subjects involved in distant education. Characterization and guidance of teachers and tutors for virtual education in library science, as well as author-tutors of learning objects, are analysed together with educational standards for the development of learning objects.

Keywords: Tutoring and virtual education; Development of Learning Objects; Educational Standards; Virtual education in library studies. 


\section{INTRODUCCIÓN}

E 1 abordaje de los problemas relacionados con la formación docente ha Etenido un importante incremento en los últimos años a raíz de los esfuerzos realizados por diversas instituciones educativas por elevar la calidad, la eficacia y la incidencia de hacerle llegar servicios educativos a una mayor población. Así, la orientación del currículum flexible, ${ }^{1}$ los requerimientos sociales de las nuevas propuestas educativas y las facilidades para construir escenarios virtuales de aprendizaje, demandan formas distintas y novedosas de comunicación e interacción entre los sujetos del acto educativo. Esto implica replantear los modelos tradicionales para la formación de docentes porque la actualidad evidencia que los diversos programas de esta naturaleza continúan reproduciendo las prácticas escolares tradicionales, y porque las políticas gubernamentales tienden a subordinar la formación docente a las necesidades de las reformas educativas en las que la participación de los docentes ha sido relegada al papel de ejecutores de tales políticas; en consecuencia, la formación docente sigue siendo una imposición desde las cúpulas antes que un espacio de propuestas educativas desde abajo. ${ }^{2}$

Por lo tanto, habrá que tener en mente que en la educación virtual el docente debe ser considerado como un elemento central para lograr el adecuado desarrollo del proceso de aprendizaje. Por ello será conveniente que él mismo participe en su formación permanente relacionada con los principios teóricos de la educación virtual, las funciones de la tutoría, la asesoría, y que adquiera y enriquezca sus habilidades en cuanto al manejo de tecnologías orientadas a la educación de esta naturaleza. No obstante, la existencia de programas de formación docente ha evidenciado, en muchos casos, problemas en su concepción debido a la falta de investigación permanente relacionada con dicha problemática, aspecto que ha provocado un escaso impacto en la práctica docente porque se ha realizado en forma rutinaria, tradicionalista y desvinculada de la relación entre docencia e investigación, lo cual impide la creatividad y la innovación en los escenarios virtuales de aprendizaje.

1 La estructura curricular flexible es aquella en la que la enseñanza tiende hacia la ciencia-acción como forma de consolidar a largo plazo una práctica reflexiva del saber y del saber hacer. Persigue adecuar permanentemente los nuevos conocimientos a los procesos de formación, promueve la capacidad de decisión en los alumnos, fomenta el trabajo colegiado, propicia la comunicación horizontal y vertical de los contenidos, facilita la movilidad de los actores académicos, opta por un aprendizaje integral, estimula la interdisciplinariedad, la disciplinariedad y la transdisciplinariedad, etcétera. En Pedroza Flores, René. Propuesta de un modelo curricular flexible para mejorar la calidad de la formación profesional", en DEP Cuadernos para la educación superior, p. 160.

2 Bosco Hernández, Martha Diana, "Dos conceptos paradigmáticos en la formación docente, la Areté y la Bildung: Una propuesta de reflexión para la educación virtual”, en Virtual Educa 2005. (Editado en CDROM) [consultado: 27/06/06] 
Dado que la educación se inscribe dentro de un entorno social, político, económico e histórico, es necesario que los docentes participen activamente en la formulación de propuestas críticas, reflexivas y analíticas que estén de acuerdo con la disciplina en cuestión, su propia práctica profesional y su experiencia personal, lo que redundará en la transformación de su práctica profesional. Esto es así porque ha evidenciado que en los ambientes de aprendizaje virtuales se requieren acciones muy activas y contundentes relacionadas con la creación de estrategias que fortalezcan la formación de los tutores desde la perspectiva de la educación permanente, y que integren procesos que partan de una formación inicial para los nuevos cuadros académicos: formación en el servicio, capacitación y actualización interdisciplinaria; y una didáctica pedagógica y tecnológica que aproveche los medios de comunicación social para apoyar estos programas. La formación sustantiva exige una paideia y una propaideia, es decir, una conciencia cultural y política del ser del hombre en su sociedad, una conciencia que pide alcanzar el mejor estatuto de hombre posible en la mejor sociedad posible. ${ }^{3}$

Estos asuntos han sufrido una revolución en estos años debido a los siguientes aspectos: el desarrollo permanente de tecnologías para el aprendizaje en escenarios virtuales; la generación constante de propuestas educativas en línea; el requerimiento de una formación permanente tanto de autores de contenido, como de tutores y de asesores, y el interés de las instituciones educativas y de los gobiernos por apoyar su intención de ampliar la cobertura social de la educación en todos sus niveles. Tales apremios han abierto un reciente debate en diversos grupos relacionados con la educación virtual por lo que se refiera a las figuras que necesita la enseñanza de esta naturaleza, entre las que se destacan: la tutoría, la asesoría y el autor de contenidos.

No obstante el requerimiento de identificar las características y funciones de dichas figuras, en la actualidad es común que una sola de ellas realice las diversas actividades que corresponderían a cada una de ellas, situación que ocasiona, entre otros aspectos, problemas de administración escolar. Por lo anterior, en el presente documento se aborda desde distintas perspectivas la urgencia de precisar las funciones del docente involucrado en propuestas educativas virtuales a partir de las figuras arriba mencionadas y con el propósito de tener una mayor claridad de las mismas para conducir sus actividades educativas con altos niveles de calidad académica. Cabe señalar que las funciones que se explican más adelante pueden ser aplicables a cualquier disciplina que opte por la modalidad virtual, sin embargo, se privilegia a la Bibliotecología por ser uno de los propósitos del presente trabajo. 


\section{CARACTERIZACIÓN Y ORIENTACIÓN DEFINITORIA DEL DOCENTE}

EN PROGRAMAS VIRTUALES

La educación virtual ${ }^{4}$ representa un fenómeno de estudio relevante para comprender los desafíos que actualmente tiene la sociedad frente a los procesos que han generado los avances científicos y los desarrollos tecnológicos. En el campo educativo es indispensable estudiar las repercusiones que produce Internet en la educación virtual, las propuestas teóricas que se discuten actualmente respecto a interactividad y navegación, el desarrollo de objetos de aprendizaje, y las perspectivas sociales e institucionales que se esperan en este siglo que inicia de la educación basada en tecnologías de red. Al mismo tiempo la convergencia tecnológica influye en el diseño de propuestas educativas virtuales en cualquier disciplina, por lo que resulta conveniente tomar en consideración los diversos aspectos tanto de su desarrollo como de su aplicación en la educación del siglo XXI. En este contexto, el desarrollo del conocimiento y la tecnología son fenómenos relevantes que los docentes relacionados con la educación virtual en Bibliotecología y en otras disciplinas deben enfrentar.

Así, la educación de esta naturaleza exige tomar en cuenta una serie de variables con el propósito de incidir, de manera eficiente, en la comunicación educativa entre los polos humanos del aprendizaje caracterizados por docentes y estudiantes. Dicha comunicación se facilita si se apoya en la institución responsable de la propuesta educativa, el programa de estudio, el currículum académico, el perfil del egresado, el mercado laboral, el desarrollo planificado de materiales didácticos y de objetos de aprendizaje, así como su distribución a través de medios que favorezcan el aprendizaje independiente y en colaboración.

Cabe resaltar el papel del docente como elemento determinante en la comunicación de los aprendizajes debido a que facilita la adquisición de conocimiento significativo; desarrolla contenidos en muchos casos; orienta y motiva

4 En este documento se hace referencia a los siguientes términos: Educación virtual: "Método de enseñanza no presencial basado en las nuevas tecnologías de la información [...] utilizando como herramienta fundamental el soporte informático, sin prescindir ni atenuar la relación profesor-alumno, pues la comunicación se mantiene y fomenta mediante la Red", en Enciclopedia de pedagogía. España: Espasa, 2002. p.1039. Educación en línea “...conjunto de métodos, tecnologías, aplicaciones y servicios orientados a facilitar el aprendizaje a distancia a través de Internet". CORNELLA, Alfonso. "E-Learning: de la formación de los empleados al conocimiento en toda la cadena de valor", en El Profesional de la Información, Vol. 11, Núm. 1, enero-febrero 2002. p. 65. Educación a distancia: Modalidad educativa que promueve en el estudiante el aprendizaje independiente, con la mediación de materiales didácticos y de TIC; además, propicia la interacción cooperativa y colaborativa de los diferentes actores del proceso educativo, de manera síncrona y asíncrona. En Coordinación de Universidad Abierta y Educación a Distancia (CUAED), Consejo Asesor. UNAM, 2005. (documento de trabajo) 
al alumno y al grupo en la búsqueda y selección de información relevante para la propuesta educativa; evalúa el aprovechamiento académico de los alumnos; evita la deserción escolar y el rezago relacionado con la eficiencia terminal, y asesora tesis en forma presencial y en línea. Así, el tutor debe tener en mente que actualmente la educación virtual se sitúa en las tecnologías de información y comunicación, y que el ciberespacio es el escenario de comunicación interactivo y comunitario que propicia la construcción de conocimiento entre los actores del aprendizaje involucrados en procesos educativos.

No obstante lo mencionado se puede afirmar que hasta nuestros días no existe un término universalmente aceptado que permita caracterizar al docente de programas educativos virtuales; más bien, la literatura especializada hace constantemente referencia al tutor, al asesor, y al consultor. La falta de precisión léxica puede deberse al hecho de que en el amplio campo de la enseñanza virtual pueden existir diversos enfoques didácticos y comunicativos. $^{5}$

Así, en la actualidad, asesor, ${ }^{6}$ tutor, profesor y consultor son las denominaciones de mayor uso para identificar el perfil y las funciones del docente que atiende programas de educación virtual en diversas instituciones de educación superior. Desde el punto de vista definitorio al tutor se le considera como "orientador, coordinador, catalizador de inquietudes, conductor del grupo y experto en relaciones humanas". En este sentido, la tutoría se concreta a la planificación general de actividades, una formulación de objetivos y una programación específica y realista. ${ }^{8}$

En los marcos institucionales, la Universidad Javeriana de Colombia afirma que entre las estrategias de la interacción, [...] se destaca la tutoría como la forma más conocida del accionar docente en programas virtuales. ${ }^{9}$ La Universidad Nacional de Educación a Distancia (UNED) de España señala que la tutoría telemática se utiliza para que el alumno pueda trabajar con su tutor a través de la computadora en cualquier momento y lugar. ${ }^{10}$ Por su parte la Universidad Virtual del Instituto Tecnológico de Estudios Superiores de Monterrey (ITESM) determina que el tutor es un docente capacitado para

5 Villegas, José Joaquín. Elementos de interacción didáctica en la enseñanza virtual: relaciones asesor-alumno. San José C.R.: UNED, 1985. p. 11

6 Especialista que asiste o asesora a los jefes o directores y que los ayuda, orienta y aconseja a la hora de tomar decisiones [...] el asesor forma, a veces, parte de consejos asesores, órganos consultivos que en el campo de la educación asisten a directores, rectores, delegados, etcétera. Diccionario de las ciencias de la educación. México: Santillana, 1995, p. 140.

7 Diccionario de las ciencias de la educación. México: Santillana, 1995. p. 1371.

8 Ibídem, p. 1372.

9 Pontificia Universidad Javeriana. Aprender virtual. [en línea] http://www.javieriana.edu.co/cua/aprender.htm [consutada: 05/07/06]

10 UNED. Régimen académico y modelo educativo. [en línea] http://www.uned.es/webuned/areargen/info/espacio6.htm [consutada: 04/05/06] 
trabajar en forma virtual, y que cuenta con la formación adecuada y la experiencia necesaria para conducir el aprendizaje independiente. ${ }^{11}$ Asimismo la Universidad de Colima, México, ha definido las siguientes figuras: profesor es el responsable del curso, de los contenidos, de la evaluación y de los resultados del curso; el tutor de contenidos apoya el montaje de contenidos; y el tutor de comunicación funge como monitor de correo electrónico, de foros de discusión y de chats de los alumnos bajo la coordinación del profesor; y está también el tutor tecnológico que apoya las dudas y problemas tecnológicos del profesor, de los tutores y de los alumnos. ${ }^{12}$

La función de asesor y de tutor que define el Sistema de Universidad Abierta y Educación a Distancia de la Universidad Nacional Autónoma de México (UNAM-CUAED), se refieren a alguien que se dedica a impartir

educación, bajo el principio de libertad de cátedra y de investigación, para formar profesionistas, investigadores, profesores universitarios y técnicos útiles a la sociedad; organizar y realizar investigaciones principalmente acerca de temas y problemas de interés nacional, y desarrollar actividades conducentes a extender con la mayor amplitud posible los beneficios de la cultura, así como participar en la dirección y administración de las actividades mencionadas. ${ }^{13}$

Recientemente, el Consejo Asesor de la CUAED se ha dado a la tarea de redefinir las figuras del asesor y del tutor para la educación abierta y a distancia, y con relación al docente asesor menciona que el término asesor tiene su raíz etimológica en el vocablo latino assessor-ōris, de assidère, asistir, ayudar a otro. En este sentido, "es la persona que asiste o aconseja”. ${ }^{14}$ Dicha acepción está de acuerdo con las funciones que realiza el profesor calificado que fomenta el estudio independiente de los alumnos, aconseja y sugiere la orientación y el ritmo del aprendizaje tomando en consideración las características particulares de cada alumno y la especificidad de la disciplina. Es la persona que orienta y guía los procesos de aprendizaje, y propicia o fomenta el estudio independiente con el apoyo de los materiales didácticos elaborados ex

11 Universidad Virtual, ITES. Beneficios de la educación en línea; el surgimiento de nuevos roles para docentes. [en línea]

http://www.ruv.itesm.mx/portal/infouv/boletines/tintero/tintero_10/articulos/5.htm [consutada: 04/10/05]

12 Información proporcionada por Margarita Almada Navarro, la que formará parte de su tesis doctoral. [entrevistada el 10/12/05]

13 UNAM, Estatuto del Personal Académico, artículo 2o. México: UNAM, 2000

14 Diccionario de la Real Academia Española. [en línea] http://www.rae.es/.2000 [Consultado: 31/07/06] 
profeso y las tecnologías de información y comunicación. ${ }^{15}$ En cuanto a la figura del docente-tutor, se ha señalado que es la persona que orienta a los alumnos de un curso o asignatura.

Más allá del mero término y la generación en diversas instituciones educativas de figuras docentes, es conveniente que el tutor tome en cuenta que el aprendizaje vía redes de teleproceso se caracteriza por la convergencia de métodos, tecnologías, aplicaciones y servicios orientados a sustentar y facilitar tal aprendizaje vía Internet. ${ }^{16}$ Para ello la educación virtual considera como fundamentales la formación docente en el manejo de plataformas tecnológicas; desarrollo de materiales didácticos; así como de objetos de aprendizaje; la evaluación de software educativo orientado a la generación de contenidos y su distribución; y la planeación de servicios de información documental acordes con la propuesta educativa virtual. Tomando en consideración la yuxtaposición de diversas funciones docentes en las modalidades educativas a distancia y en línea, los siguientes rubros de este documento abordan de manera especial aquellas que pueden corresponder a las figuras del tutor y al autor de contenido en la educación virtual en Bibliotecología.

\section{LA TUTORÍA EN LA EDUCACIÓN VIRTUAL EN BIBLIOTECOLOGÍA}

La función del tutor en la educación virtual es considerada como el elemento nuclear para el buen desarrollo de los procesos de aprendizaje orientados a la construcción de conocimiento en los alumnos. Así, los tutores que orientan el aprendizaje y los sujetos que aprenden, se mantienen en una comunicación de interactividad que genera nuevas formas de comunicar contenidos, conocimiento e información a través de los diversos medios tecnológicos.

A este respecto Amador señala que

del texto escrito y los audiovisuales didácticos, a los programas vía satélite y a las redes informatizadas, la convergencia de los audiovisuales, la informática y las telecomunicaciones trajo como consecuencia la creación de redes telemáticas. ${ }^{17}$

15 UNAM. Coordinación de Universidad Abierta y Educación a Distancia, Consejo Asesor. Las figuras académicas participantes en el Sistema Universidad abierta y Educación a Distancia: Propuesta para su consideración en el Nuevo Estatuto del Personal Académico de la UNAM. (Documento de trabajo, 2005). p.6

16 Cornella, Alfonso. "E-Learning: de la formación de los empleados al conocimiento en toda la cadena de valor", en El Profesional de la Información, Vol. 11, Núm. 1, enero-febrero 2002. p. 65.

17 Amador Bautista, Rocío."Nuevos procesos educativos en el medio digital”, en El medio digital en el siglo XXI..., [Editado en CD-ROM]. Trabajo presentado en el XVIII Coloquio Internacional de Investigación Bibliotecológica. 
Así, el uso de tales medios se concreta a partir de las posibilidades de generar escenarios virtuales de aprendizaje que se configuran con base en la convergencia tecnológica en red y les dan valor y sentido a los sujetos del aprendizaje virtual.

Las recientes formas del aprendizaje virtual reclaman que se investigue sobre la concepción del modelo normativo del tutor, ${ }^{18}$ modelo que debe considerar, entre otros aspectos, las funciones generales que caracterizan a este tipo de tutoría, entre las cuales se encuentran las siguientes:

1. La tutoría como función pedagógica se apoya en el principio de que el aprendizaje es un proceso que se realiza en el interior del sujeto y que en esa dinámica se generan eventos que favorecen la secuencia de este aprendizaje.

2. La tutoría como acción motiva, ayuda y refuerza procesos de aprendizaje independiente.

3. La tutoría como valor debe aceptar las diferencias individuales de cada alumno ya que éstas pueden condicionar el ritmo particular de los aprendizajes.

4. La tutoría debe otorgarse en cualquier momento.

5. La tutoría como orientadora debe promover el uso convergente de múltiples medios para estimular el interés del alumno frente a su situación de aprendizaje. Asimismo debe propiciar la combinación de medios, métodos y recursos documentales para evitar una excesiva dependencia de los alumnos, lo que puede fomentar el estudio independiente.

Al mismo tiempo en la educación virtual el tutor debe tener en mente que las Tecnologías de Información y Comunicación (TIC) presentan alternativas competitivas en comunicación, en la entrega de materiales didácticos y en la recuperación de información complementaria a los aprendizajes. Las tecnologías permiten generar sistemas rizomáticos que

... a diferencia de los sistemas arborizantes de conocimiento, donde se plantea un transcurso lógico de un principio hasta un fin (en los contenidos de aprendizaje), los sistemas rizomáticos de conocimiento plantean un escenario de permanente búsqueda hacia diversas direcciones, en donde el trascurso es fragmentario y es también indefinido: las múltiples posibilidades de seguir, detenerse o regresar a

18 Elementos basados en: Fanny Locker Dupuy, Cruz Manuel Guédez y IUT Cumaná. "Las competencias profesionales del tutor académico en la educación virtual”, en Universitas 2000. Vol. 16 no. 3. 1992. 
un punto, enriquecen la toma de decisiones de la persona que manipula estas secuencias de puntos sin un fin preconcebido. ${ }^{19}$

En este sentido, cabe resaltar que la educación virtual se apoya en el hipertexto y en las tecnologías de red, las cuales ofrecen diversas posibilidades de comunicación con los alumnos, controles académicos versátiles, desarrollo de materiales didácticos basados en estructuras hipertextuales, videoconferencias, servicios de correo electrónico, bibliotecas digitales y sistemas de metadatos. Asimismo, esta educación acentúa la generación de servicios de diseminación selectiva de la información bibliográfica vía telecomunicaciones y la transferencia de documentos digitales de apoyo a los aprendizajes a distancia.

Por tanto, se puede señalar que las tecnologías de información y comunicación ofrecen diversas y ricas posibilidades para el desarrollo de propuestas educativas virtuales y de contenidos educativos con fines de docencia e investigación. Un logro importante de este tipo de propuestas se relaciona con un alto grado de control administrativo, la difusión masiva vía Internet de cursos en diversos niveles, facilidades de interacción entre alumnos y tutores y la posibilidad de consultar vía Internet diversas fuentes documentales.

En este contexto conviene que la tutoría tenga en mente que las propuestas sobre educación virtual en Bibliotecología partan de un acercamiento teórico y metodológico sustentado en el cuerpo de conocimientos de la disciplina, de su vinculación con los requerimientos informativos y culturales de la sociedad de la que se trate, de la calidad sustentada en la enseñanza interdisciplinaria y multidisciplinaria, y de la investigación científica. Así, puede ser determinante el desarrollo de tareas de gestión educativa que incluyan aprendizaje innovador, la formación de tutores académicos, la preparación de autores de contenido, la participación de tutores pedagógicos, los expertos en diseño gráfico, y los administradores de aulas virtuales.

Con base en estos señalamientos se puede mencionar que atañe a la Bibliotecología procurar un proceso permanente de formación de tutores debido a que con la penetración de tecnologías de información y comunicación en la disciplina, los conocimientos en el área serán cada vez más dinámicos, situación que necesariamente redundará en los procesos de formación y en el ejercicio de la profesión. Los cambios sustantivos que se han dado en los modelos sociales y económicos, necesariamente inciden en la educación bibliotecológica, y por ello cabe resaltar la conveniencia de que la disciplina Filosofía y Letras, Coordinación de Universidad Abierta y Educación virtual, 2004. p.49 
diversifique sus campos de conocimiento, desarrolle nuevas alternativas de formación tomando en cuenta los avances del conocimiento en la disciplina, enfrente las demandas de los mercados laborales, incorpore el uso de TIC en la producción y la difusión de nuevos conocimientos, innove sus métodos y técnicas de aprendizaje, y continúe desarrollando investigación básica y aplicada, todo lo cual naturalmente redundará en la figura del tutor.

Ya no hay duda de que la tutoría en los sistemas educativos virtuales en Bibliotecología y otras disciplinas será esencial debido a que se ha comprobado que una asesoría deficiente produce en los alumnos perdida de motivación e interés, aspecto que generalmente desemboca en la deserción. En resumen, las funciones relevantes del tutor en lo relativo a programas de educación virtual en Bibliotecología abarcan una amplia gama de actividades como las que a continuación se describen.

Funciones de tutoría

\begin{tabular}{|c|}
\hline Dominar los contenidos elaborados para la enseñanza de la disciplina bibliotecológica \\
\hline Participar en el diseño instrumental de módulos de aprendizaje virtuales orientados a dicha disciplina. \\
\hline Colaborar en la creación de aulas virtuales de apoyo al aprendizaje de la bibliotecología. \\
\hline $\begin{array}{l}\text { Participar en el desarrollo de contenido educativo tomando en cuenta las características de la enseñaza en } \\
\text { Bibliotecología. }\end{array}$ \\
\hline $\begin{array}{l}\text { Planear y preparar la asesoría a distancia para los módulos que conformen el programa orientado a la } \\
\text { enseñanza de la disciplina. }\end{array}$ \\
\hline Aplicar los principios teóricos de la educación virtual en la enseñanza. \\
\hline $\begin{array}{l}\text { Guiar a los alumnos en las particularidades del estudio independiente tomando en cuenta los contenidos } \\
\text { educativos de apoyo a la enseñanza de la disciplina. }\end{array}$ \\
\hline Propiciar la interacción dinámica en el aprendizaje a distancia. \\
\hline $\begin{array}{l}\text { Inducir el estudio cooperativo y en colaboración a distancia abordando, por ejemplo, problemas relevantes } \\
\text { o de actualidad relacionados con la Bibliotecología. }\end{array}$ \\
\hline $\begin{array}{l}\text { Posibilitar la adquisición de conocimiento significativo que redunde tanto en el conocimiento que adquieren } \\
\text { los alumnos como en su ejercicio profesional. }\end{array}$ \\
\hline $\begin{array}{l}\text { Fomentar y orientar en los alumnos la selección de información relevante complementaria a los materiales } \\
\text { didácticos y a los objetos de aprendizaje, induciéndolos a utilizar bibliotecas digitales y otras fuentes } \\
\text { especializadas. }\end{array}$ \\
\hline $\begin{array}{l}\text { Promover la utilización de los medios de comunicación de mayor utilidad para apoyar el aprendizaje virtual } \\
\text { de la disciplina. }\end{array}$ \\
\hline $\begin{array}{l}\text { Organizar foros virtuales de discusión en donde participen especialistas y alumnos a distancia sobre temas } \\
\text { bibliotecológicos por medio de preguntas, opiniones y reflexiones. }\end{array}$ \\
\hline Orientar en línea a los alumnos en forma individual a lo largo del proceso de aprendizaje. \\
\hline Propiciar el desarrollo de destrezas para la compresión de materiales didácticos u objetos de aprendizaje \\
\hline Proponer estrategias didácticas para el aprendizaje en línea de la Bibliotecología. \\
\hline $\begin{array}{l}\text { Realizar pruebas de evaluación virtual, cuya finalidad será la de ayudar al estudiante a sistematizar su } \\
\text { aprendizaje y a hacerlo sentirse orientado y motivado al comprobar su aprovechamiento. }\end{array}$ \\
\hline Investigar sobre la docencia en Bibliotecología en escenarios virtuales de aprendizaje \\
\hline
\end{tabular}


Funciones del autor de contenido en las que debe participar el tutor

Diseñar y elaborar materiales didácticos como guías de estudio, antologías, libros ex profeso, manuales, cuadernos de ejercicios, tomando siempre en cuenta las teorías sobre el aprendizaje.

Diseñar y elaborar objetos de aprendizaje basados en estándares educativos y teorías del aprendizaje utilizando herramientas tecnológicas ex profeso.

Tener a la mano destrezas para el manejo de software educativo para el diseño de material didáctico y objetos de aprendizaje apropiados para la enseñanza virtual de la Bibliotecología.

Como se puede observar, las funciones y actividades del tutor en programas de educación virtual pueden ser copiosas y variadas; la intención de lo anteriormente enlistado pretende que tales funciones resuelvan las necesidades y deficiencias de los alumnos relacionadas con sus aprendizajes a través de la revisión y seguimiento de su avance formativo a distancia. El tutor deberá realizar el seguimiento de las actividades de autoaprendizaje orientando y controlando los resultados y sugiriendo actividades que tiendan a desarrollar las destrezas de los alumnos en ambientes virtuales de aprendizaje, contextualizando los fenómenos bibliotecológicos. Asimismo, ayudará a los alumnos a tomar conciencia de las particularidades del aprendizaje virtual de la disciplina bibliotecológica induciendo en el alumno la adopción de una posición de aprendizaje activo que lo lleve a una continua búsqueda de preguntas y el planteamiento de problemas a través de la interactividad académica virtual, siempre tomando en cuenta los problemas inherentes a la Bibliotecología. También deberá motivar y reforzar las actitudes del alumno hacia el autoaprendizaje a distancia partiendo del supuesto de que una de las funciones de la Bibliotecología como una disciplina humanística es otorgar servicios de información acordes con los características de la sociedad a la que sirve.

Con todo lo anterior, el tutor tiene la oportunidad de ser el enlace entre la institución educativa, los alumnos, el currículum, el programa, los materiales didácticos, los objetos de aprendizaje y las plataformas tecnológicas. Por lo tanto, se encuentra en una situación privilegiada para identificar las principales áreas de dificultad de los sistemas de comunicación y de los contenidos didácticos. Un buen tutor toma en cuenta que en la comunicación educativa, el solo uso de cualquier técnica didáctica o tecnología no es garantía de éxito, puesto que el proceso de comunicación a distancia depende de diversos factores ligados a cuestiones de índole subjetiva: el interés permanente de comunicación por parte del tutor, los estados de ánimo y las condiciones ambientales y particularidades tecnológicas en las que se encuentren los sujetos del acto educativo a distancia.

Sin embargo, en la comunicación educativa puede resultar muy atractivo para los sujetos del aprendizaje el uso de aulas virtuales debido a que el 
correo electrónico y los foros de discusión facilitan la creación de ambientes de pertenencia a determinada comunidad disciplinaria o a un ambiente digital de comunicación en múltiples direcciones: alumno-institución, alumno-alumno, alumno-tutor, además de que pueden darse varios tipos de participación: enviar-recibir mensajes, o bien simplemente consultar su contenido didáctico o realizar sus actividades de aprendizaje.

Así, con el uso de las TIC, un rubro teórico importante de la tecnología educativa se refiere a la formación integral del tutor que atenderá programas a distancia, lo que implicará incidir en una pedagogía que sea “... autoinstructiva, interactiva, personalizadora y que a su vez, incida sobre el aprendizaje, los medios y la metacognición". ${ }^{20}$ En este sentido, el tutor de programas virtuales en Bibliotecología debe también participar en la vida colegiada de la institución a la que pertenece, conocer las características de la educación de adultos, y participar en la elaboración de contenidos didácticos que respondan a las necesidades de los estudiantes tomando en cuenta las particularidades de operación social de los sistemas bibliotecarios, bibliográficos y de información.

Al mismo tiempo, la interactividad y la navegación en red son rasgos de gran interés para la tutoría, debido a su novedad y a los pocos avances que se han logrado al respecto hasta nuestros días. Por ello los tutores en escenarios virtuales deben analizar detenidamente los problemas que pueden causar dichos fenómenos en el proceso de aprendizaje vía redes de teleproceso. Por eso es necesario que el tutor que participe en propuestas educativas virtuales en Bibliotecología se involucre, además de lo ya señalado, en las siguientes tareas:

- Manejar los ambientes tecnológicos que contemplen el conjunto de actividades de aprendizaje, experiencias y recursos documentales de apoyo.

- Evaluar la infraestructura de comunicación remota que facilite la interacción no presencial entre tutores y alumnos a través de actividades como discusiones en tiempo real y en tiempo diferido.

- Evaluar contenidos de sitios web relacionados con los temas que se aborden en los módulos de aprendizaje específicos.

- Comprobar que la plataforma tecnológica responda a los requerimientos de la propuesta educativa virtual cuidando: las facilidades para el ingreso; consulta y transmisión de contenidos didácticos; la interacción

20 Antonio J. Colom C., en su obra Pedagogía tecnológica para la educación virtual, propone demostrar porqué la pedagogía que él llama tecnológica es el constructo teórico más pertinente y el que mejor se adapta para el desarrollo de la educación virtual. 
en tiempo-espacio a través de aplicaciones de correo electrónico, foros de discusión, pizarrón electrónico, grupos de discusión, chat y videoconferencia para producir bienes colectivos e intercambiar ideas en torno a los fenómenos de estudio; la realización y control de la evaluación del alumno, del tutor y del programa académico, la instrumentación de la administración escolar, y las facilidades para realizar flujos de información en diversos formatos digitales como aplicaciones World-Wide Web, FTP (File Transfer Protocol), .TEX, etcétera).

- Colaborar en el diseño de ambientes de aprendizaje que contemplen el conjunto de actividades de aprendizaje, así como las experiencias y recursos documentales de apoyo a la reconstrucción de conocimientos.

- Sugerir herramientas de comunicación remota que faciliten la interacción no presencial entre tutores y alumnos.

- Programar seminarios virtuales para el intercambio de una información que propicie la reflexión sobre temas específicos en donde la comunicación entre los alumnos se realice desde distintos lugares y sea mediada vía sistemas de telecomunicación, programas y reglas para ordenar la información que se requiera transferir y la que resulte de la discusión.

El tutor también deberá tener en mente la generación exponencial de información en Internet, que puede ser de mucha utilidad para desarrollar actividades de aprendizaje específicas, tal información puede ser un apropiado complemento del contenido didáctico que se haya desarrollado ex profeso para determinada propuesta educativa virtual. En este sentido el tutor debe conducir al alumno para que éste responda a preguntas como las siguientes:

- ¿Cómo se localiza en línea la información de utilidad para la propuesta educativa específica?

- ¿Cómo se realiza el análisis para seleccionar la información de mayor relevancia a los temas de estudio?

- ¿Cómo se incide en el descubrimiento de información relevante adicional a los materiales didácticos y a los objetos de aprendizaje desarrollados para determinado módulo de aprendizaje?

- ¿Cómo se logra que esa información redunde en conocimiento significativo para el sujeto del aprendizaje? y, en su momento, ¿para qué y cómo recrearla?

En definitiva, todo programa relacionado con la formación de tutores para la educación virtual en Bibliotecología debe contemplar un tutor reflexivo, 
que aplique la observación, problematice, tome decisiones, planifique, investigue e intervenga no sólo en el proceso de enseñanza; sino en el contexto en el que éste se desenvuelve. Parte del análisis de su práctica se orienta en la comprensión de su realidad y en la generación de propuestas concretas y realizables. En consecuencia, un proceso tutoral de calidad contempla una visión holística en la planeación, una actitud empática e interés y compromiso de los actores del aprendizaje en cuanto a las acciones a desarrollar. Por lo tanto el tutor debe contar con una sólida formación profesional, actualización interdisciplinaria, experiencia en investigación a partir de líneas concretas, interés por la innovación educativa, capacidad pedagógica y dominio de la actividad tutorial en escenarios virtuales de aprendizaje. ${ }^{21}$

Se advierte que las funciones del tutor pueden ser múltiples y en diversos casos se yuxtaponen con determinadas actividades que desempeñan los maestros de programas de educación presencial, sin embargo, cabe resaltar que la educación virtual exige funciones que no necesariamente le son inherentes a la educación presencial, como se habrá notado en párrafos precedentes. No obstante, la necesidad de contar con un referente que identifique las funciones propias de las figuras del tutor y del autor de contenido educativo, en su momento lo ideal sería que dichas figuras tuvieran movilidad tanto en programas de educación virtual como en propuestas educativas basadas en otras modalidades educativas, de tal manera que un solo perfil pudiera cubrir a la vez los requerimientos tanto de tutoría como del desarrollo de contenido.

Sin embargo, lograr tal perfil requiere un trabajo intenso de cuerpos colegiados que comprendan cabalmente lo que implica la tutoría y la autoría de contenido en programas educativos virtuales y se interesen en las distintas modalidades educativas como alternativas para incidir en la preparación de amplios sectores sociales. Lo anterior beneficiaría y facilitaría, entre otros aspectos, la evaluación del trabajo docente para su promoción o para nuevas contrataciones. Además de lo señalado con anterioridad, se consideró conveniente reflexionar en torno al desarrollo de objetos de aprendizaje.

\section{El Autor DE ObJetos DE APRENDIZAJE}

Desde una perspectiva histórica la educación a distancia ha mostrado que los contenidos didácticos representan uno de los elementos necesarios en la formación de esta naturaleza porque son el enlace de comunicación educativa de

21 Alvarado Hernández, Víctor y Rosalba Romero Escalona. Los aspectos cualitativos de la tutoría en educación superior. UNAM, Escuela Nacional de Estudios Profesionales Aragón. (Documento preparado para el Primer Encuentro Nacional de Tutoría en Colima, Colima, p. 16. 
mayor importancia entre quien enseña y quien aprende. Así, el desarrollo de contenido ha exigido que los autores asuman ciertas posturas teóricas al elaborar tales contenidos. Comprender lo que implica educar a distancia ha sido un requisito indispensable para desarrollar contenidos que cumplan con los requerimientos de la comunicación vía redes de teleproceso, la interacción, la navegación y el diálogo académico remoto entre los actores del aprendizaje.

Desde hace algunos años se observa que la tecnología de información y comunicación ha enriquecido las maneras de realizar dichas tareas a través de la convergencia tecnológica de las telecomunicaciones y la informática, cuya función es incidir en acciones relacionadas con el desarrollo de software educativo, sistemas basados en multimedios, hipertexto e hipermedia con el fin de enriquecer las maneras de realizar los aprendizajes a distancia. El uso intensivo de dichas tecnologías se relaciona con los objetivos y los temas de estudio, por lo que la identificación y el tratamiento de los mensajes académicos representan tareas de gran valor en tanto que se requiere discriminarlos y estructurarlos tomando en cuenta marcos psicopedagógicos y las características particulares de los medios portadores del contenido educativo.

El empleo de modelos dirigidos al desarrollo de contenido didáctico presupone también acudir al conocimiento y a la aplicación de un conjunto de principios normativos derivados de las teorías del aprendizaje y con el cual se pretende hacer más eficiente la elaboración de contenidos. Una característica relevante respecto a los materiales didácticos que se emplean en la educación a distancia es su alto grado de estructuración pedagógica debido a que su finalidad consiste en reforzar los aprendizajes. Por ello, la búsqueda de un conocimiento significativo implicaría tomar en consideración que los contenidos educativos deben tener un ordenamiento lógico y facilitar, con fundamento en bibliografías complementarias, la búsqueda por parte del alumno de información adicional sobre los temas de estudio.

Por lo anterior, se debe procurar hacer llegar al estudiante un discurso académico accesible a su aprendizaje que lo incite a la reflexión, a la síntesis y a la generación de conclusiones significativas respecto a los temas de estudio, lo que resulta un reto para todo autor de contenido. Así, el aprendizaje en escenarios virtuales tiene mayor valor cuando el alumno es capaz de crear ideas, desarrollar y resolver problemas, y adquirir destrezas que lo hagan útil a la sociedad y al grupo profesional al que pertenezca.

Por lo tanto, se percibe que el autor de contenido no se crea tan fácilmente porque su desarrollo consume un tiempo considerable, exige la especialización en los temas en que habrá de desarrollar y precisa destrezas en el manejo de tecnologías para el soporte de contenido. Cuando se trata de desarrollar objetos de aprendizaje, la situación se complica porque se requiere además 
de conocimiento teórico, lo cual exige el desarrollo de material didáctico, que dicho autor se familiarice con la nuevas propuestas relacionadas con los estándares educativos y su repercusión en la educación virtual del siglo XXI.

Por esta razón y tomando en cuenta que existe una amplia bibliografía respecto con el desarrollo de materiales didácticos, en este rubro se privilegia lo relacionado al desarrollo de objetos de aprendizaje por ser un fenómeno de mucha actualidad, debido a que a la educación virtual le está dando mucha importancia en el marco mundial en tanto que pone de manifiesto la necesidad de contar con grupos especializados y multidisciplinarios para abordar tareas relacionadas con el desarrollo de este tipo de objetos.

Así, para elaborar de objetos de aprendizaje es determinante tomar en consideración que la calidad académica y pedagógica que se pretenda lograr en los productos finales, así como su alcance y complejidad estarán influidas por los recursos humanos y la disponibilidad financiera; y también habrá que contar con especialistas de distintas disciplinas puedan garantizar el logro de contenidos de mayor calidad y versatilidad. Así, en función de la propuesta educativa, el autor de objetos de aprendizaje procederá a su desarrollo. Lo anterior indica que éste deberá tener, o en su caso desarrollar, diversas competencias, pues requiere estructurarse un perfil que no necesariamente ha requerido en otros momentos el desarrollo de material didáctico para la educación a distancia; tal perfil demanda lo siguiente:

1. Capacidad para identificar problemáticas educativas relevantes a partir de una determinada disciplina y para proponer soluciones encaminadas a resolver situaciones educativas específicas de la disciplina en cuestión en los marcos regional, nacional, local o global.

2. Destrezas para el desarrollo de contenido tomando en consideración, cuando proceda, tratamientos multi, inter y transdisciplinarios.

3. Capacidad y conocimiento para incorporar contenidos de aprendizaje en los propios objetos de aprendizaje.

4. Conocimiento de una pedagogía autogestiva que conduzca a la creación de estrategias de aprendizaje a distancia orientadas a lograr reflexión, síntesis y capacidad conclusiva en los alumnos.

5. Capacidad para crear objetos de aprendizaje que sean reutilizados a través de redes locales de amplio espectro y en diversos contextos, carreras o áreas de conocimiento.

6. Interés para evaluar las prácticas docentes y gestionar innovaciones psicopedagógicas y tecnológicas pertinentes al desarrollo y uso de objetos de aprendizaje.

7. Apertura a los aportes que se hacen desde otras disciplinas a la propia. 
8. Capacidad para hacer interactuar al usuario del objeto de aprendizaje con el objeto mismo por medio de recursos digitales. Y aptitud para adecuar los contenidos para ser utilizados por diferentes estudiantes en diferentes contextos.

9. Capacidad de traducir en las instrucciones psicopedagógicas los contenidos que se presenten en el objeto de aprendizaje. ${ }^{22}$

El desarrollo de estas competencias por parte de los autores de objetos de aprendizaje implica abordar su formación desde perspectivas que no necesariamente ha exigido la educación a distancia tradicional debido a que el valor de mayor relevancia que puede tener el desarrollo de objetos de aprendizaje radica en que su contenido pueda ser reutilizado en entornos de red, que es lo que les incorpora un valor agregado inédito. Sin embargo, es muy conveniente que dicho autor tenga un conocimiento profundo sobre los estándares educativos involucrados en el desarrollo y uso de los objetos de aprendizaje.

\section{ESTÁNDARES EDUCATIVOS EN EL DESARROLLO DE OBJETOS DE APRENDIZAJE}

Los estándares educativos orientados a la educación virtual tienen como fin proponer un conjunto de elementos tecnológicos que armonicen plataformas y objetos de aprendizaje con el propósito de facilitar su desarrollo, el intercambio de contenido y su reutilización. Por lo tanto, algunos propósitos de dichos estándares se dirigen a los siguientes aspectos:

- Durabilidad. La tecnología desarrollada con base en estándares debe prever la obsolescencia de los contenidos de aprendizaje.

- Interoperabilidad. El intercambio de contenidos debe contemplar una amplia variedad de Learning Management System (LMS).

- Accesibilidad. El monitoreo académico de los alumnos debe ser permanente.

- Reusabilidad. El mayor valor de los cursos y de los objetos de aprendizaje reside en que puedan ser reutilizados con diferentes herramientas y en distintas plataformas. ${ }^{23}$

22 Cf. Navarro Cendejas, José y Luis Fernando Ramírez Anaya. Objetos de aprendizaje: formación de autores con el modelo redes de objetos. México. UdeGVIRTUAL, 2005. p.43.

23 Romero, Daniel [y otros]. "Gateway para el reciclaje de sistemas e-learning que no cumplen con SORM". En Primer Congreso Virtual Latinoamericano de Educación a Distancia. 23 de marzo al 4 de abril de 2004.

http://www.ateneonline.net/datos/65_03_Romero_Daniel.pdf [Consultada 10-05-2004] 
Con relación a dichos asuntos, Castellanos ha señalado que en el e-Aprendizaje, los metadatos describen e identifican los contenidos educativos para que éstos puedan ser encontrados, ensamblados y enviados [...] El contenido se divide en piezas pequeñas de información llamadas objetos de aprendizaje las cuales pueden ser reutilizadas, adaptadas a los perfiles específicos de los alumnos y a los objetivos del aprendizaje. ${ }^{24}$

En cuanto a las características de mayor relevancia de los objetos de aprendizaje se destacan las siguientes: se pueden representar en unidades de contenido digital, refuerzan conceptos, principios o procedimientos, son durables al evitar la obsoletización del contenido, son inter-operables en cuanto se contemplan facilidades para su intercambio a través de LMS, accesibles para monitorear el desempeño de alumnos en forma permanente, reutilizables con el apoyo de diferentes herramientas y plataformas tecnológicas, lo cual indica que deben ser flexibles y adaptables al desarrollo de distintas versiones a partir del objeto de aprendizaje primario. En cuanto a la estructura básica de su contenido se contempla que habrá de incorporar objetivo, contenido propiamente dicho y parámetros de evaluación. Al mismo tiempo es conveniente que el contenido de los objetos de aprendizaje se sustente en teorías pedagógicas: teoría del procesamiento de la información, constructivismo, etcétera. En cuanto a los medios portadores del objeto de aprendizaje se identifican principalmente audiovisuales, digitales y multimedia.

Cabe destacar que en la actualidad los estándares educativos de mayor aceptación son los siguientes: IEEE Standard for Learning Objet Metadato (IEE1484.12), que contiene especificaciones para la generar de objetos de aprendizaje utilizando metadatos; IEEE Draft Standard for XML Binding For Learnig Object Metadata, contempla directrices para el marcado de contenido en lenguaje XML; Advanced Distributed Learning (ADL) que se generó para desarrollar un software orientado a OA; Instructorial Management System Global Learning Consortium (IMS) se refiere a normas que orientan el desarrollo de plataformas virtuales; Entreprise Specification (IMS) que se utiliza para gestionar cursos vía Internet; Question ETest Specification (IMS) que consiste en un módulo para evaluar aprendizajes.

El Learning Management System (LMS) contempla las especificaciones tecnológicas orientadas hacia una plataforma para servidores Internet e Intranet; Learning Content Management Systems(LCMS) contempla elementos para generar el repositorio de objetos de aprendizaje, herramientas para el desarrollo de OA, herramientas de publicación, herramientas de colaboración

24 Castellanos Coutiño, Carlos Alberto. "Panorama general de los sistemas de educación a distancia”. Ponencia en el Primer Congreso Virtual Latinoamericano de Educación a Distancia: LatinEduca2004.com

http://www.ateneonline.net/datos/19_01_castellanos_carlos.pdf [Consultada: 10/03/2005] 
para la generación de OA, interfaz dinámica para la entrega de OA y elementos para el monitoreo de los actores del aprendizaje; Sharable Content Object Reference Model (SCORM) son especificaciones para el desarrollo de software, el empaquetado y la entrega de objetos de aprendizaje; Advanced Distributed Learning (ADL) contiene normas para la publicación de contenidos derivados de objetos de aprendizaje. En el esquema de la página derecha se agrupan los estándares educativos de mayor relevancia y son de hecho, aquellos en los que los autores de objetos de aprendizaje requieren estar actualizados con el propósito de conocer su manejo, alcance y utilidad en el desarrollo de OA y los requerimientos de éstos para ser intercambiados en diversas plataformas tecnológicas con fines de reutilización en distintos escenarios geográficos con el fin de apoyar aprendizajes relacionados con disciplinas afines.

Se advierte que en el conjunto de estándares mencionados con anterioridad es factible identificar aquellos que están orientados hacia la definición de unidades de aprendizaje y los que apoyan la sistematización de repositorios en red. Respecto a la definición de unidades de aprendizaje se tiende a utilizar las especificaciones y recomendaciones de IMS debido a su especialización y por ser uno de los estándares de uso generalizado. El sistema IMS contempla una serie de propuestas y la caracterización de implantaciones suficientemente extensas como por ejemplo la que se incluye como parte del modelo SCORM. ${ }^{25}$ Con relación a los estándares orientados a la construcción de repositorios en red, éstos aún no están suficientemente desarrollados por lo que es necesario definir los elementos estructurales de los repositorios distribuidos en red y dar una definición lógica del repositorio en cuanto a su arquitectura interna y funciones de implantación.

La definición de un estándar de interoperatividad debe permitir la interconexión de repositorios distribuidos a partir de metadatos para objetos de aprendizaje, con el Núcleo de Dublín o MARC 21, aspecto que hasta nuestros días se encuentra en etapa de investigación y experimentación. La teoría y la escasa experiencia que se tiene al respecto ha mostrado que los estándares educativos orientados al desarrollo de objetos de aprendizaje demanda especificaciones más abiertas y flexibles para incorporar metadatos y establecer principios para construir una taxonomía relacionada con éstos a fin de sustentar principios teóricos y tecnológicos para su desarrollo. Al mismo tiempo, se hace visible el apremio por incorporar propuestas bibliotecológicas en

25 Cf. Prototipo de patrimonio público de recursos educativos basados en una red institucional y un repositorio distribuido de objetos de aprendizaje. Participantes: Ma. Elena Chan Núñez. Universidad de Guadalajara, Jorge Martínez Peniche. DGSCA-UNAM, Rafael Morales Gamboa. Instituto de Investigaciones Eléctricas, Víctor G. Sánchez Arias. Laboratorio Nacional de Informática Avanzada A.C. Reporte de Investigación, 2004. (documento interno de trabajo) p.14 


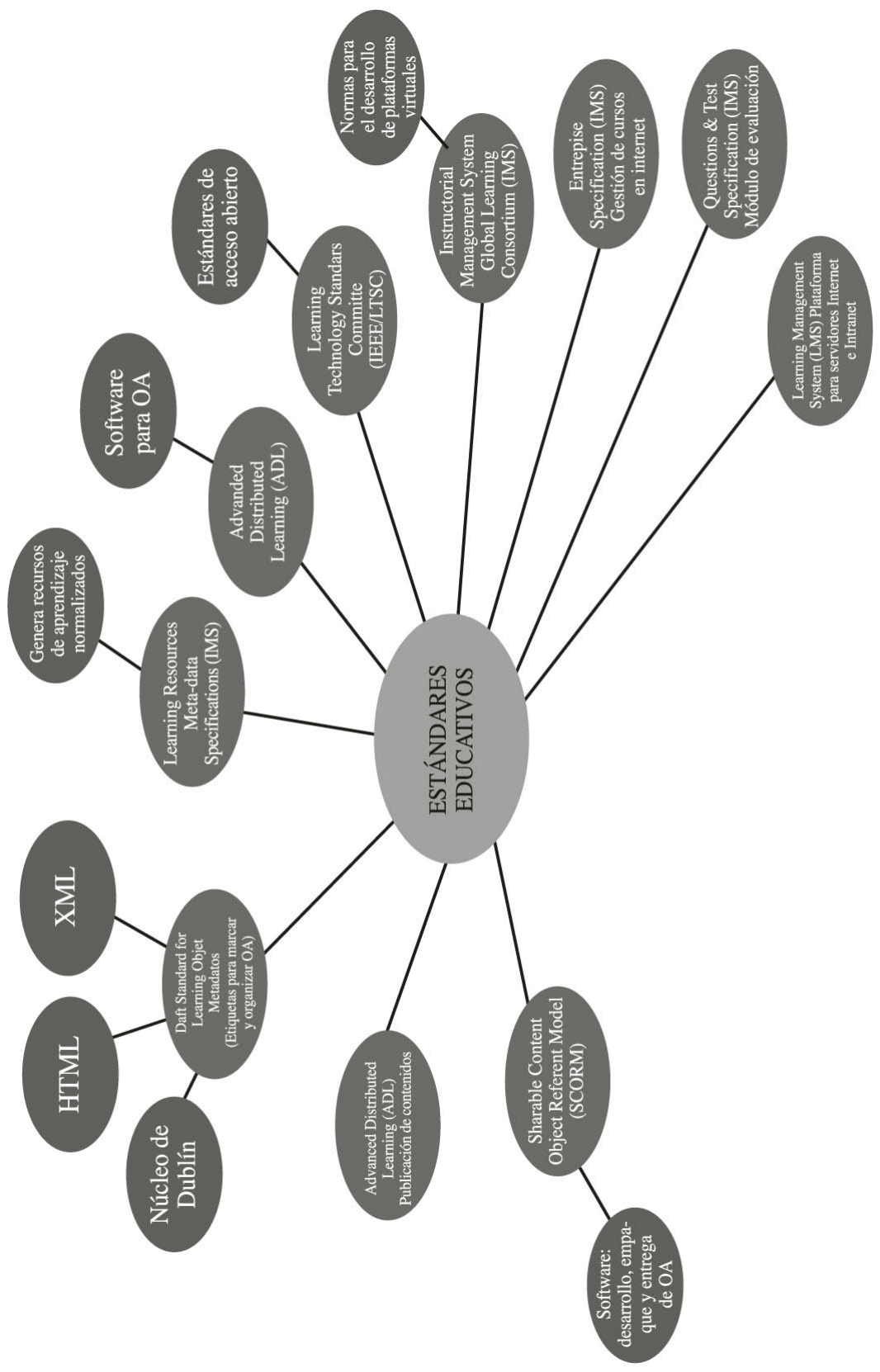


materia de organización documental a partir de la construcción de sistemas de metatados y bibliotecas digitales de objetos de aprendizaje.

El autor de contenido debe tener en mente que el objeto de aprendizaje, como fenómeno emergente, se encuentra en constante evolución debido a los avances psicopedagógicos y a los desarrollos permanentes de las tecnologías involucradas. Así, el objeto se genera, se adapta y se modifica de acuerdo con las necesidades educativas o las imposiciones de la innovación tecnológica, lo cual ha motivado que éste sea visto como un objeto único que presenta nuevas realidades de desarrollo, uso y aplicación en el aprendizaje virtual de las disciplinas para el siglo XXI.

Con lo anterior se intenta evidenciar la exigencia de la formación de autores de contenido ya que sin duda las escuelas que tienen programas en bibliotecología de América Latina y el Caribe interesadas en la educación virtual, se beneficiarían al reutilizar los objetos de aprendizaje desarrollados en otras escuelas, con lo que al mismo tiempo se fomentaría la cooperación interinstitucional al conjugar intereses comunes en el desarrollo de contenidos educativos, lo que en su momento, podrían ser de mucha utilidad en cualquier modelo educativo y no sólo en el virtual.

\section{A MANERA DE CONCLUSIONES}

En lo que se refiere a propuestas de educación virtual habrá que considerar que la comunicación entre asesores, tutores, alumnos, contenidos y medios reclama marcos pedagógicos que involucren aprendizaje significativo, innovador y permanente, así como el fomento del aprendizaje individual, cooperativo y la interacción con especialistas y comunidades académicas en escenarios virtuales.

La timidez de los programas que se han generado hasta el momento para la formación docente en Bibliotecología, no han aportado hasta nuestros días lo que está exigiendo la educación virtual debido a la escasa vinculación entre investigación y docencia.

Identificando las actividades fundamentales del tutor y las funciones inherentes al autor de objetos de aprendizaje en propuestas educativas virtuales, es factible asegurar formas efectivas de tutoría de esta modalidad educativa en el más amplio sentido y a la vez que específicas. Asimismo se puede contribuir a fortalecer los reglamentos institucionales conducentes a orientar el trabajo de los cuerpos colegiados respecto al ingreso, la permanencia, la evaluación del desempeño de la tutoría y la promoción de los docentes que desarrollen tareas relacionadas con programas de educación virtual. 
La Bibliotecología se enfrenta a La necesidad de desarrollar nuevas alternativas de formación tomando en cuenta los avances del conocimiento de la disciplina; las demandas de los mercados laborales y los desarrollos tecnológicos orientados a la producción y la difusión de nuevos conocimientos; y a la exigencia de reformular sus métodos y técnicas de enseñanza-aprendizaje y de continuar desarrollando la investigación básica y la aplicada. En consecuencia un punto medular tendrá que ser la propuesta de programas permanentes para la formación de tutores y autores de contenido que preparen programas de educación virtual.

La evaluación de la actividad docente requiere de marcos referenciales que indiquen las funciones específicas de la tutoría en programas de educación virtual, aspecto que convendría que formara parte de la normatividad institucional con el propósito de facilitar la evaluación docente. Esto es un asunto no resuelto y en consecuencia, preocupante para las instituciones educativas debido al constante incremento de programas de educación virtual; situación que, por cierto, afecta a la Bibliotecología.

En la educación virtual, el tutor y el autor de contenido deben tener en mente que las tecnologías presentan alternativas competitivas en la comunicación, la entrega de contenidos educativos, y en la recuperación de información complementaria a los aprendizajes. En este sentido, puede resaltarse que la estructura informativa de la educación virtual se basa en el hipertexto. Así, las tecnologías de red ofrecen diversas posibilidades de comunicación con el alumno, controles académicos versátiles, y un desarrollo de materiales didácticos y de objetos de aprendizaje basados en estructuras de hipertexto y multimedia, y también se acentúa la generación de flujos de información digital de apoyo complementario a la educación virtual. En este contexto la comunicación educativa, la interacción, el diálogo, el modelo docente de aprendizaje, el material didáctico, los objetos de aprendizaje, la asesoría y la tutoría en línea constituyen la base sobre la cual se sustenta el éxito o fracaso de cualquier propuesta educativa de esta naturaleza.

La innovación tecnológica relacionada con objetos de aprendizaje ha generado un conjunto de estándares educativos para que tales objetos puedan ser desarrollados, organizados, recuperados y transmitidos vía redes de teleproceso y reutilizados por los actores del aprendizaje virtual. El hecho de que tales estándares no incluyan de manera específica aspectos psicopedagógicos, más que una debilidad puede verse como una oportunidad para que tutores y autores de contenido educativo propongan o generen teorías que incidan en aprendizaje significativo en escenarios virtuales en beneficio de la bibliotecología y de los alumnos a distancia.

El discurso del objeto de aprendizaje lo genera el autor de contenido y adquiere, un mayor sentido cuando se encuentra en línea y lo utilizan los alumnos 
a distancia como una herramienta que estructura contenidos flexibles, manipulables, reutilizables, que tiene con otros objetos y que son trasferibles a través de Internet debido a que puede ligarse en diversos sentidos. Los elementos que lo constituyen hacen de él un objeto inacabado, por lo que propicia su reutilización obedeciendo los objetivos de la propuesta educativa específica. Asimismo, el desarrollo de objetos aprendizaje rebasa los recursos digitalizados puesto que implica cambios de paradigma en la forma de concebir el proceso educativo, el cual puede tener implicaciones y aplicaciones en distintos ámbitos educativos, su potencial depende sobre todo de la visión y amplitud de la mirada de quienes los conciban y quienes los utilicen.

En suma, la Bibliotecología como disciplina debe considerar la innovación educativa para insertar a tutores, autores de objetos de aprendizaje, alumnos y egresados, en la competitividad que exige la internacionalización de la educación superior y la movilidad de profesionales tomando en consideración, entre otros aspectos, el desarrollo cultural y la cooperación académica en un mundo global. No es aventurado afirmar que los procesos educativos virtuales de las diversas disciplinas aseguran su éxito cuando existe calidad académica en sus docentes, sus contenidos educativos, sus bibliotecas y en la habilidad didáctica de los primeros en el uso apropiado de los medios de aprendizaje sustentados en tecnologías de información y comunicación.

\section{BiBLIOGRAFÍA CONSULTADA}

Alvarado Hernández, Víctor y Rosalba Romero Escalona. Los aspectos cualitativos de la tutoría en educación superior. UNAM, Escuela Nacional de Estudios Profesionales Aragón. (Documento preparado para el Primer Encuentro Nacional de Tutoría en Colima). 18 p.

Amador Bautista, Rocío. "Nuevos procesos educativos en el medio digital", en El medio digital en el siglo XXI: retos y perspectivas para los bibliotecólogos, investigadores, educadores y editores. México: UNAM, CUIB, [Editado en CD-ROM, 2001]. Trabajo presentado en el XVIII Coloquio Internacional de Investigación Bibliotecológica.

Barrón Soto, Héctor S. La educación en Línea y el texto didáctico. México: UNAM, Coordinación de Universidad Abierta y Educación a Distancia: Facultad de Filosofía y Letras, 2004. 100 p.

Bisquerra Alzina, Rafael, Coord. La práctica de la orientación y la tutoría. Barcelona: CISSPRAXIS, 2002.289p.

Bosco Hernández, Martha Diana. "Dos conceptos paradigmáticos en la formación docente, la Areté y la Bildung: Una propuesta de reflexión para la educación virtual", en Virtual Educa 2005. (Editado en CDROM) [consultado: 27/06/06] 
Castellanos Coutiño, Carlos Alberto. Panorama general de los sistemas de educación a distancia. Ponencia en el Primer Congreso Virtual Latinoamericano de Educación a Distancia:LatinEduca2004.com

http://www.ateneonline.net/datos/19_01_castellanos_carlos.pdf [Consultada: 10/05/06]

Coordinación de Universidad Abierta y Educación a Distancia. Educación a distancia (aproximación definitoria). México: CUAED, UNAM, 2005. (documento de trabajo del Consejo Asesor de la CUAED).

Collis, B. Tele-learning in a digital world: the future of distance learning. London: International Thompson Computers Press, 1996. $651 \mathrm{p}$.

Contenidos e-learnig. En e-learnig América Latina. La Revista Digital de e-Learnig en América Latina. Año1, número 8, noviembre de 2004. [en línea]

http://www.elearnigamericalatina.com/edicion/noviembre/index.php [consultada: 06/07/06]

Colom Cañellas, Antonio J. "Pedagogía tecnológica para la educación virtual”, en Los nuevos escenarios educativos y las transformaciones tecnológicas / comp. Patricia Ávila Muñoz. México: ILCE, 1998. p. $25-40$

Contreras M. Rita. Reflexiones en torno al uso de la tecnología de la información en el terreno educativo. En Soluciones Avanzadas: tecnologías de información y estrategias de negocios jun. 1997, vol. 5, no. 46, p. $10-15$

Cornella, Alfonso. "e-Learning: de la formación de los empleados al conocimiento en toda la cadena de valor", en El Profesional de la Información, Vol. 11, Núm. 1, enero-febrero 2002. pp. 65-68.

Diccionario de las ciencias de la educación. México: Santillana, 1995. 1431p. Pontificia Universidad Javieriana. Aprender virtual. [en línea] http://www.javieriana.edu.co/cua/aprender.htm [consutada: 04/06/06]

Diccionario de la Real Academia Española. [en línea] http://www.rae.es/.2000 [Consultado: 21/11/05]

Draft standard for learning object metadata. Sponsored by the Learning technology Standards Committee Piscataway, N. J. : IEEE Standards

Department, 2002 [en línea] http://ltsc.ieee.org/wg12/files/LOM_1484_12_1_v1_Final_Draft. pdf [consultada: 14/05/05]

The Dublin Core Initiative. [en línea] http://purl.oclc.org/metadata/Dublin core/ [consultada: $16 / 02 / 05$ ]

Dupuy, Fanny Locker, Cruz Manuel Guédez y IUT Cumaná. "Las competencias profesionales del tutor académico en la educación virtual”, en Universitas 2000. Vol. 16 no. 3. 1992. 
E-learning: soluciones de e-learning. Formación a Distancia [en línea]. <http://e-learning.bankhacker.com/ > [consultada: 08/05/06]

Institute of Electronic and Electronics Engineers, Inc. IEEE 1484.12.12002 Draft Standard for Learnig Objet Metadata. New York: IEEE, 2002. 44p..

Iriarte Navarro, Leonel [et al.]. Generación de una biblioteca de objetos de aprendizaje (LO) a partir de contenidos preexistentes. Revista de Educación a Distancia, no. 2, 2005 [ en línea ] http://www.um.es/ead/M2 [consultado; 14/04/05]

Laorden, Cristina, Elena García y Salvador Sánchez. Integrando descripciones de habilidades cognitivas en los metadatos de los objetos de aprendizaje estandarizados, 2005 [en línea ] http://spedece.uah.es/papers/Laorden_Final.pdf. [14/04/06]

Lever-Duffy, Judy. Teaching and learning with technology / Judy Lever-Duffy, Jean B. McDonald, Al P. Mizell. - Boston: Pearson Education, Inc., c2003. xxi, 439 p.

Locker Dupuy, Fanny, Cruz Manuel Guédez y IUT Cumaná. Las competencias profesionales del tutor académico en la educación virtual. En Universitas 2000. Vol. 16 no. 3. 1992.

Loyo Varela, C. y V.G. Sánchez. La telemática y la educación a distancia. En Soluciones Avanzadas. Vol. 4(24) 1995. [en línea].

$<$ http://www.lania.mx/nivel2/divulgacion.html> [consultada: $15 / 0505]$

Navarro Cendejas, José y Luis Fernando Ramírez Anaya. Objetos de aprendizaje: formación de autores con el modelo redes de objetos. México. UdegviRTuAL, 2005. 61p.

Olea Deserti, Elia. "Relevancia del tutor en los programas a distancia", en Apertura: Revista de innovación educativa. Año 5(2) 2005. pp. 6-19.

Pedroza Flores, René. Propuesta de un modelo curricular flexible para mejorar la calidad de la formación profesional. DEP Cuadernos para la educación superior, p. 160

Pontificia Universidad Javieriana. Aprender a distancia. [en línea] http://www.javieriana.edu.co/cua/aprender.htm [consutada: 05/07/06]

Prototipo de patrimonio público de recursos educativos basados en una red institucional y un repositorio distribuido de objetos de aprendizaje. Participantes: Ma. Elena Chan Núñez. Universidad de Guadalajara, Jorge Martínez Peniche. DGSCA-UnAM, Rafael Morales Gamboa. Instituto de Investigaciones Eléctricas, Víctor G. Sánchez Arias. Laboratorio Nacional de Informática Avanzada A.C. Reporte de Investigación, 2004. 38p. (documento interno de trabajo)

Reunión de la Comisión Académica de Objetos de Aprendizaje. (Guadalajara, Jal. 4 de julio 2002). [en línea]

$<$ http://www.cudi.org/ob_ap.html > [Consultada:10/10/03] 
Romero, Daniel y otros]. Gateway para el reciclaje de sistemas e-learning que no cumplen con SCORM. En Primer Congreso Virtual Latinoamericano de Educación a Distancia 2004. [en línea] http://www.ateneonline.net/datos/65_03_Romero_Daniel.pdf [Consultada: 10/05/06]

Rovira, Cristofol. La orientación a objetos en el diseño de bipertextos para la enseñanza-aprendizaje, 1999 [ en línea ]

http://www.ucm.es/info/multidoc/multidoc/revista/num8/rovira.html [consultado: 02/03/05]

Subotovsky, Santiago. Consideraciones básicas para su proyecto de desarrollo de contenidos, 2004 [ en línea ]

http://www.elearningamericalatina.com/edicion/noviembre1_2004/ na_1.php [consultada: 14/04/05]

Tele-learning: the challenge for the third millennium / Edited by Don Passey, Mike Kendall. Norwell, Massachusett: Kluwer Academic Publishers, 2002. xxiii, 372 p.

Uned. Régimen académico y modelo educativo. [en línea] http://www.uned.es/webuned/areargen/info/espacio6.htm [consutada: 04/05/06]

UNAM. Coordinación de Universidad Abierta y Educación a Distancia, Consejo Asesor. Las figuras académicas participantes en el Sistema Universidad abierta y Educación a Distancia: Propuesta para su consideración en el Nuevo Estatuto del Personal Académico de la UNAM. Documento de trabajo, 2005)

UNAM, Estatuto del Personal Académico, artículo 2o . México: UNAM, 2000

Universidad Virtual, ITES. Beneficios de la educación en línea; el surgimiento de nuevos roles para docentes. [en línea]

http://www.ruv.itesm.mx/portal/infouv/boletines/tintero/tintero_10/articulos/5.htm [consutada: 04/05/06] Universidad Virtual, ITES. Beneficios de la educación en línea; el surgimiento de nuevos roles para docentes. [en línea]

http://www.ruv.itesm.mx/portal/infouv/boletines/tintero/tintero_10/articulos/5.htm [consutada: 03/07/06]

Villegas, José Joaquín. Elementos de interacción didáctica en la enseñanza virtual: relaciones asesor-alumno. San José C.R.: UNED, 1985. $67 \mathrm{p}$.

Zapata Ros, Miguel. Secuencia de contenido y objetos de aprendizaje. RED. Revista de Educación a Distancia, no. 2, 2005 [ en línea ] http://www.um.es/ead/red/M2//zapata47.pdf [consultada: 14/04/06] 